\title{
Relation Between Gas Absorptivities and Emissivities*
}

\author{
D. Olfe and S. S. Penner \\ Daniel and Florence Guggenheim Jet Propulsion Center,
California Institute of Technology, Pasadena, California \\ (Received June 20,1958)
}

$I^{\mathrm{N}}$ previous theoretical studies, ${ }^{1-4}$ expressions have been obtained between infrared gas absorptivities and emissivities for several descriptions of vibration-rotation bands. Although these expressions provide a good correlation of the (limited) available experimental data, it is of interest to supplement the earlier results by the relation corresponding to absorption of radiation by molecular vibration-rotation bands composed of randomly distributed dispersion lines with arbitrary intensity probability distribution function.

The absorptivity $\left(\alpha_{a b}\right)$ of a gas at temperature $T_{g}$ and of optical depth $X$ for blackbody radiation at temperature $T_{s}$ is given by the relation

$\alpha_{a b} \simeq \frac{1}{\sigma T_{s}^{4}} \Sigma\left[\bar{R}_{i}^{0} \omega_{i}\left(T_{s}\right)\right] \int_{\Delta \omega_{i}\left(T_{\theta}\right)}\left\{1-\exp \left\{-\left[P_{\omega}\left(T_{g}\right) X\right]\right\}\right\} d \omega$,

where $\sigma$ represents the Stefan-Boltzmann constant, $\bar{R}^{0} \omega_{i}$ is the blackbody radiancy at the wave number corresponding to the center of the $i$ th band and $P_{\omega}$ is the spectral absorption coefficient of the gas. For the statistical model corresponding to a wave number interval of effective width $\Delta \omega_{i}\left(T_{q}\right)$,

where

$$
\alpha_{a b} \simeq \frac{1}{\sigma T_{s}^{4}} \sum_{i}\left[\bar{R}^{0} \omega_{i}\left(T_{s}\right)\right]\left\{1-\exp \left[-\frac{A_{i}\left(T_{g}\right)}{d_{i}^{*}\left(T_{g}\right)}\right]\right\} \Delta \omega_{i}\left(T_{g}\right),
$$

$$
\bar{A}_{i}=\int_{0}^{\infty} P\left(\bar{S}_{i} / S\right) A_{L i} d\left(\bar{S}_{i} / S\right)
$$

is the weighted mean value of the line absorption $A_{L i}$ computed with respect to the normalized intensity probability distribution function $P\left(\bar{S}_{i} / S\right)$, and $d_{i}{ }^{*}$ is the mean line spacing for lines in the $i$ th band. For dispersion lines of half-width $b$, the line absorption is $A_{L} \simeq S X$ for $S X / 2 \pi b<2 / \pi$ and $A_{L} \simeq 2(S b X)^{\frac{1}{2}}$ for $(S X / 2 \pi b)$ $>2 / \pi$. For the linear region $1,3,4$

$$
\alpha_{a b}=\left(\frac{T_{s}}{T_{b}}\right) \epsilon\left(T_{s}, X\right) .
$$

On the other hand, for the square root region,

$$
\bar{A}_{i}=2\left(b_{i} X\right)^{\frac{1}{2}} \int_{0}^{\infty} P\left(\bar{S}_{i} / S\right) S^{\frac{1}{2}} d\left(\bar{S}_{i} / S\right)=\operatorname{constan} t\left(\bar{S}_{i} b_{i} X\right)^{\frac{1}{2}}
$$

Hence, assuming that $\bar{S}_{i}=\alpha_{i} /\left(\Delta \omega_{i} / d_{i}{ }^{*}\right)$ where $\alpha_{i}$ is the integrated absorption for the $i$ th band, $\Delta \omega_{i}(T)$ proportional to $T^{\frac{1}{2}}, \alpha_{i}$ proportional to $T^{-1}, b_{i}$ proportional to $T^{-\frac{1}{2}}$, and

we obtain

$$
d_{i}^{*}\left(T_{0}\right) / d_{i}^{*}\left(T_{s}\right)=\left(\frac{T_{s}}{T_{g}}\right)^{\eta}
$$

$$
\frac{\bar{A}_{i}\left(T_{g}\right)}{d_{i}^{*}\left(T_{g}\right)}=\mathrm{constant} \frac{\left[\bar{S}_{i}\left(T_{s}\right) b_{i}\left(T_{s}\right) X\left(T_{s} / T_{g}\right)^{2-\eta}\right]^{\frac{1}{2}}}{d_{i}^{*}\left(T_{s}\right)}
$$

whence Eq. (2) becomes

$$
\alpha_{a b} \simeq\left(T_{g} / T_{s}\right)^{\frac{1}{\epsilon}} \in\left[T_{s}, X\left(T_{s} / T_{g}\right)^{2-\eta}\right] .
$$

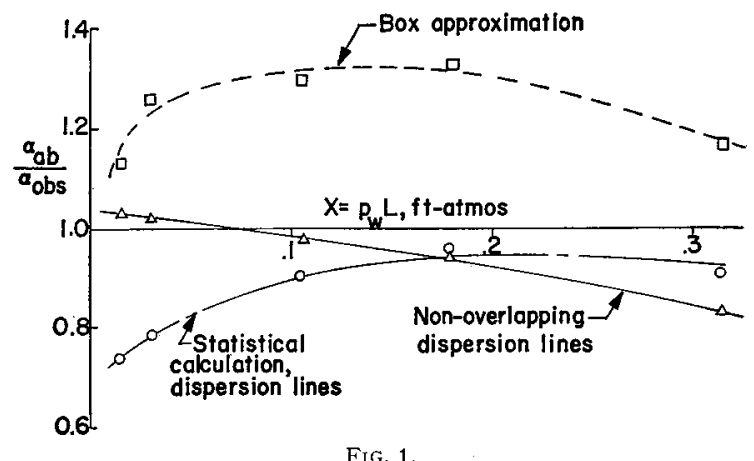

For water vapor $\eta$ is about unity $y^{4-6}$ and Eq. (5) reduces to

$$
\alpha_{a b} \simeq\left(T_{b} / T_{s}\right)^{\frac{1}{2}} \in\left[T_{s}, X\left(T_{s} / T_{g}\right)\right]
$$

which is very nearly the same as Hottel's empirical formula for water vapor, viz.,

$$
\alpha_{H}=\left(T_{J} / T_{s}\right)^{-0.45} \epsilon\left[T_{s}, X\left(T_{s} / T_{g}\right)\right] .
$$

For well overlapped and "just overlapping" lines it has been shown previously ${ }^{1-4}$ that

$$
\alpha_{a b}=\left(T_{g} / T_{s}\right)^{\frac{1}{s}} \in\left[T_{s}, X\left(T_{s} / T_{g}\right)^{\frac{3}{2}}\right]
$$

whereas, for nonoverlapping dispersion lines with $\eta=1,1,3,4$

$$
\alpha_{a b}=\left(T_{g} / T_{s}\right) \epsilon\left[T_{s}, X\left(T_{s} / T_{g}\right)^{2}\right] .
$$

A representative comparison of observed and calculated results is shown in Fig. 1 for water vapor. Reference to Fig. 1, and to data for larger values of $X$ which are not plotted, shows that Eq. (6) gives a fair representation for $X$ greater than about 0.1 or $0.2 \mathrm{ft}$-atmos; Eq. (7) holds for very small values of $X$ and also for $X$ greater than about $0.5 \mathrm{ft}$-atmos; Eq. (8) applies for $X$ less than about $0.2 \mathrm{ft}$-atmos. These results are consistent with the approximations involved in the derivation of Eqs. (6) to (8). Equation (6) should apply when the absorption is dominated by dispersion lines in the square root region irrespective of the extent of overlapping between lines.

* Supported by the Office of Naval Research, U. S. Navy, under Contract Nonr-220 (03). NR 015401.

1 A. Thomson, Ph.D. thesis, California Institute of Technology, Pasadena, June, 1958.

2 S. S. Penner and A. Thomson, J. Appl. Phys. 28, 615 (1957).

3 Thomson, Hooker, and Penner, J. Appl. Phys. 30, 124 (1959).

4 S. S. Penner, Ouantitative Molecular Spectroscopy and Gas Emissivities (Addison-Wesley Publishing Company, Inc., Reading, Massachusetts) (to be published).

A. Thomson, "An approximate analytic expression for the engineering emissivity of water vapor," Technical Note No. 4, Gruen Applied Science aboratory, Pasadena, California, April, 1957. western University Press, Evanston, Illinois, 1957), pp, 151-173. 